\title{
Nramp Transfection Transfers Ity/Lsh/Bcg-Related Pleiotropic Effects on Macrophage Activation: Influence on Oxidative Burst and Nitric Oxide Pathways
}

\author{
C. Howard Barton, Simon H. Whitehead, and \\ Jenefer M. Blackwell \\ University of Cambridge Clinical School, Department of Medicine, \\ Addenbrooke's Hospital, Cambridge, United Kingdom
}

\begin{abstract}
Background: The Ity/Lsh/Bcg gene on mouse chromosome 1 regulates priming/activation of macrophages for antimicrobial and tumouricidal activity. A candidate gene expressed in macrophages has been identified by positional cloning and full-length sequence analysis, and encodes the Natural resistance-associated macrophage protein (Nramp). In this study, we have tested the hypothesis that the Nramp gene corresponds to Ity/Lsh/Bcg. Materials and Methods: In vitro transfection was used to introduce the resistant allele into the macrophage cell line RAW 264.7 derived from the recessive susceptible $\mathrm{BALB} / \mathrm{c}$ mouse strain. Expression of the transgene was monitored on the background of the endogenous susceptible allele by allele-specific oligonucleotide hybridization

Results: Expression of the transgene correlated with three $L s h^{\mathrm{r}}$-associated lipopolysaccharide/interferon- $\boldsymbol{\gamma}$ -
\end{abstract}

regulated macrophage activation phenotypes: respiratory burst, nitrite release, and uptake of L-arginine. Endogenous and stimulated L-arginine fluxes were inhibitable with the radical scavengers nordihydroguaiaretic acid and butylated hydroxyanisole. The mitochondrial electron transport inhibitors, rotenone and thenoyltrifluoroacetone, inhibited respiratory burst, and rotenone suppressed L-arginine flux, implying that mitochondrial-derived oxygen radicals are important mediators in Nramp-regulated signal transduction pathways.

Conclusions: These data provide the first direct evidence that Nramp is the product of the Ity/Lsh/Bcg gene, and are consistent with the hypothesis that the many pleiotropic effects of this gene on macrophage activation may all derive from the requirement for mitochondrial generation of oxygen radicals for intracellular signaling.

\section{INTRODUCTION}

The murine macrophage resistance gene Ity/Lsh/ $B c g$ was first described for its role in in vivo regulation of Salmonella typhimurium (Ity) (1), Leishmania donovani $(L s h)(2)$, and mycobacterial infections including Mycobacterium bovis (Bcg) (3). Subsequent studies of macrophage function using congenic mouse strains demonstrated that the gene influences lipopolysaccharide (LPS)/interferon- $\gamma$ (IFN $\gamma)$-regulated macrophage prim-

Address correspondence and reprint requests to: Jenefer $M$. Blackwell, Department of Medicine, Level 5, Addenbrooke's Hospital, Hills Road, Cambridge CB2 2QQ, United Kingdom. ing/activation, exerting a wide range of pleiotropic effects in vitro (reviewed in Refs. 4-9) including regulation of the $\mathrm{C}-\mathrm{X}-\mathrm{C}$ neutrophil chemoattractant $\mathrm{KC}$, interleukin $1 \beta$ (IL- $1 \beta$ ), and inducible nitric oxide (NO) synthase (iNOS) mRNA; surface major histocompatibility complex (MHC) class II, 5' nucleotidase, and AcM.1 antigen expression; and tumor necrosis factor $\alpha$ (TNF $\alpha$ ) and NO release, L-arginine flux, respiratory burst (RB), and tumouricidal activity. For $L$. donovani, the final effector mechanism for kill by resistant macrophages activated in vitro relies on the TNF $\alpha$-dependent sustained generation of NO (10). In vivo, the effects of $I t y / L s h / B c g$ on early T 
cell-independent regulation of all three infections $(1-3)$, as well as on the later development of an IFN $\gamma$ generating CD4-positive $\mathrm{T}$ cell response (11), presumably reflect synergy between the many pleiotropic effects of the gene on macrophage function. Hence, while no one of these phenotypes is measurable as more than a modest quantitative effect in vitro, the effect in vivo is dramatic producing 2-3 log-fold differences in parasite numbers as early as 7-8 days postinfection (12). The wide range of pleiotropic effects of the gene measurable in vitro, some of which can be monitored as early as 30 min after LPS stimulation of resistant macrophages (13), suggest a role for the gene in early signaling pathways leading to macrophage activation.

Recently, positional cloning identified a partial cDNA encoding the Natural resistance-associated macrophage protein (Nramp) as a candidate for the murine resistance gene Ity/Lsh/Bcg (14). In addition to its position in the genome, candidacy was based (I) on the macrophagerestricted expression of Nramp, and (2) on the presence of a common mutation in all susceptible mouse strains $(14,15)$. Confirmation by transfection/transgenesis has not previously been reported. The more recent isolation of a full-length cDNA (16) now provides the basis for transfection analysis, and hence for determining the underlying molecular mechanism for the action of this gene. Unfortunately, the deduced amino acid sequence provided no definitive clues as to how the gene might work. Nramp encodes a hydrophobic, polytopic integral membrane protein with a conserved transport motif (14) and an $\mathrm{N}$-terminal cytoplasmic region encoding a Pro/Ser rich SH3 binding domain (16). Identity across the conserved transport motif with the nitrate transporter CrnA of Aspergillus nidulans led to the earlier hypothesis (14) that Nramp might be involved in the direct delivery of nitrates across the phagolysosome membrane. Although the acid environment of the phagolysosome could mediate conversion of nitrates to nitrites and antimicrobial NO, this hypothesis does not adequately account for the many pleiotropic effects of the gene. The subsequent observation that both murine (9) and human (17) Nramp genes show $53-58 \%$ sequence similarity with two yeast proteins, SMF1 and SMF2, which regulate protein translocation across the mitochondrial membrane, suggests a more fundamental mechanism related to mitochondrial function. It is of particular interest, for example, that the apoptotic and gene-inductive effects of
$\mathrm{TNF} \alpha$ have recently been shown to involve the mitochondrial generation of reactive oxygen intermediates (ROI) required for intracellular signaling and activation of the transcription factor, NFkB (18). Demonstration that mitochondrially derived ROI are involved in the signaling pathway required for induction of $I t y / L s h / B c g-r e g u-$ lated macrophage phenotypes would provide support for further investigation of a mitochondrial function.

In the present study, gene transfer experiments have been carried out in vitro to determine whether the candidate molecule, Nramp, can influence macrophage priming/activation using RB activity, nitrite release, and uptake of $\mathrm{L}$-arginine as markers of resistance. This is the first study to demonstrate expression of a resistant phenotype with gene transfer of the Nrampresistant allele. Use of radical scavengers and mitochondrial electron transport inhibitors also provide evidence that Nramp function might lie in the pathway leading to the mitochondrial generation of ROI required for intracellular signaling.

\section{MATERIALS AND METHODS}

\section{Preparation of Expression Constructs}

The isolation and characterization of the fulllength Nramp cDNA ( $\lambda 8.1)$ has been described previously (16). The insert from this clone was prepared by restriction with EcoRI and XhoI and ligated between the EcoRI and SalI sites of the pBabe vector (19), which contains a linked puromycin resistance marker under the control of the SV40 early promoter. A susceptible allele construct was prepared by removing a fragment from $\lambda 8.1$ using HindIII and MscI endonucleases, flanking the mutation in the second membrane spanning domain, and replacing it with a similarly digested fragment obtained by reverse transcriptase-PCR amplification from macrophagederived RNA from susceptible BALB/c mice. Recombinant clones were subjected to sequence analysis to ensure that the only mutation present was the susceptibility-associated Gly $\rightarrow$ Asp transition at position $169 \mathrm{bp}$ in the full-length sequence, and appropriate inserts cloned into the expression vector as described.

\section{Transfection Protocols and Analysis of Recombinant Clones}

The macrophage cell line selected for transfection was BALB/c-derived RAW 264.7 (20), since 
it can be easily transfected by electroporation and stable lines established (21). To confirm that the endogenous copy of Nramp encoded a susceptible allele, a RTPCR fragment was generated over the susceptibility-associated mutation and sequenced. Electroporation was performed essentially as described (21) with $10 \mu \mathrm{g}$ plasmid DNA at $900 \mu \mathrm{F}$ and 300 volts $(750 \mathrm{~V} / \mathrm{cm})$ with a resistance of 481 ohms. Following electroporation cells were plated in $10 \mathrm{ml}$ of DMEM containing $10 \%$ fetal calf serum and 20 mM HEPES in a $90-\mathrm{mm}$ petri dish for $48 \mathrm{hr}$ to recover, prior to the addition of puromycin to a final concentration of $4 \mu \mathrm{g} / \mathrm{ml}$. Colonies were left to appear over a 2-week period, isolated using cloning rings and expanded. Some colonies were recloned by limiting dilution into 96-well dishes. Clones were routinely maintained in media containing puromycin except during experimental procedures.

\section{Allele-Specific Oligonucleotide Hybridization}

Individual clones were monitored for transgene expression by an allele-specific oligonucleotide hybridization (ASO) assay. RNA was prepared by extraction with guanidine isothiocyanate/acid phenol and subjected to DNAase I treatment to remove any residual DNA containing expression plasmid DNA. RNA $(1 \mu \mathrm{g})$ primed with random hexamers was reverse transcribed and amplified (100 ng) with Nramp-specific primers spanning the susceptibility-associated mutation (CAT CTC TAC TAC CCC AAG GTG C; TTG CGC AAA CCA TAG TTA TCC). Products were denatured in alkali at $100^{\circ} \mathrm{C}$ and spotted onto nylon membranes. Filters were probed in duplicate with end-labeled 15-mers corresponding to the R/S sequence (CTGTGGG G/A CGGTGTA) at $37^{\circ} \mathrm{C}$ in $6 \times$ SSPE, $0.5 \%$ SDS, $100 \mu \mathrm{g} / \mathrm{ml}$ salmon sperm DNA. Filters were washed in conditions such that each oligonucleotide would only hybridize to its specific sequence: $1 \times$ SSC, $0.1 \%$ SDS at $42^{\circ} \mathrm{C}$ for the susceptible; $50^{\circ} \mathrm{C}$ for the resistant 15 -mers.

\section{Measurement of Macrophage Respiratory Burst}

Transfected cells were harvested from subconfluent $90-\mathrm{mm}$ plates by scraping, counted, and viability assessed in the presence of trypan blue. Cells were seeded at $10^{5}$ /well in $100 \mu \mathrm{l}$ of medium in 96-well plates and allowed to adhere for
$1 \mathrm{hr}$ prior to stimulation with $25 \mathrm{ng} / \mathrm{ml}$ LPS for 24 or $30 \mathrm{hr}$ as indicated. Respiratory burst (RB) was measured as superoxide anion production following $\mathrm{l} \mathrm{hr}$ triggering with phorbol myristate acetate (PMA, $500 \mathrm{ng} / \mathrm{ml}$; Sigma Chemical Co., Poole, Dorset, United Kingdom) in the presence of nitrobluetetrazolium (NBT, $2 \mathrm{mg} / \mathrm{ml}$; Sigma). The reaction was terminated by removing the media and thorough washing with methanol to remove excess NBT. The plate was allowed to air dry and the precipitated formazan produced by interaction of superoxide anion with NBT was solubilized with $120 \mu \mathrm{l}$ of $2 \mathrm{M} \mathrm{KOH}$ and $140 \mu \mathrm{l}$ DMSO (22). The plate was read at $630 \mathrm{~nm}$ and blanked on medium including NBT and cells without PMA.

\section{Macrophage Nitrite Production}

Nitrite levels were measured as the stable end product of inducible nitric oxide synthase (iNOS)-generated nitric oxide (NO) using 24- or 30 -hr supernatants from IFN- $\gamma(25 \mathrm{U} / \mathrm{ml}) / \mathrm{LPS}(25$ $\mathrm{ng} / \mathrm{ml}$ ) activated macrophages mixed in equal volumes with freshly prepared Griess reagent ( $1 \%$ sulfanilamide, $0.1 \%$ naphthylethylenediamine hydrochloride, and $2.5 \%$ orthophosphoric acid in water) and absorbances at $570 \mathrm{~nm}$ determined. The concentration of nitrite was determined from a standard curve from 0 to $200 \mu \mathrm{M}$ with sodium nitrite.

\section{Normalization for Cell Density}

For both RB and nitrite experiments, replica plates were prepared and assayed colorimetrically $(0.5 \%$ crystal violet in $20 \%$ methanol) to allow normalization for cell number. After they were stained, the wells were thoroughly rinsed in water and air dried, and absorbances were read at $540 \mathrm{~nm}$.

\section{Assay for Uptake of L-Arginine}

L-arginine uptake was measured $6 \mathrm{hr}$ after stimulation with $25 \mathrm{U} / \mathrm{ml}$ IFN $\gamma$ and $25 \mathrm{ng} / \mathrm{ml} \mathrm{LPS}$. The culture medium employed contained 0.4 $\mathrm{mM} \mathrm{L}$-arginine excluding any contribution from serum. Pilot experiments demonstrated that the uptake of $\left[{ }^{3} \mathrm{H}\right]$-L-arginine $(0.25 \mu \mathrm{Ci}$, specific activity $58 \mathrm{Ci} / \mathrm{mmol}$ ) from $10^{5}$ cells was linear over a $\mathrm{l}-\mathrm{hr}$ time period at $37^{\circ} \mathrm{C}$. In all subsequent experiments, cells were pulsed for 30-45 min (i.e., during the 5 th-6th hour after IFN $\gamma /$ LPS stimulation). The incubation was terminated by 
removing the media and washing the adherent cells three times in phosphate buffered saline (PBS) containing $10 \mathrm{mM}$ unlabeled L-arginine. Cells were lysed in $50 \mu \mathrm{l}$ of $1 \%$ SDS and counted in $5 \mathrm{ml}$ of aquasol II (DUPONT-NEN).

\section{Inhibition Studies}

L-arginine uptake experiments were performed in the presence of the radical scavengers nordihydroguaiaretic acid $(0-40 \mu \mathrm{M})$ and butylated hydroxyanisole $(0-400 \mu \mathrm{M})$. Respiratory burst and L-arginine uptake experiments were also carried out in the presence of the mitochondrial electron transport inhibitors rotenone $(0-40$ $\mu \mathrm{M}$; inhibits complex I $\rightarrow$ ubiquinone) or thenoyltrifluoroacetone (TTFA; $0-400 \mu \mathrm{M}$; inhibits complex II $\rightarrow$ ubiquinone). Concentrations of inhibitors were based on previous studies (18) examining the role of mitochondrially derived ROI on apoptosis and the gene-inductive effects of TNF $\alpha$ in fibroblasts in vitro, and were not observed to have toxic effects on the RAW 264.7-derived transfectant lines. Cells were allowed to adhere to microtitre wells for $1 \mathrm{hr}$ prior to a 1-hr pretreatment with drugs before addition of activation agents for appropriate time periods.

\section{RESULTS}

\section{ASO Assays}

The rationale for all functional experiments performed here was that the resistant allele of the Lsh gene is dominant (23) and that its transfer would mediate effects measurable on the background of the endogenous susceptible allele. Sequencing across the region of the Gly $\rightarrow$ Asp susceptibility-associated mutation confirmed that the BALB/c-derived $\left(L s h^{s}\right)$ RAW 264.7 recipient cell line carried an Asp codon at amino acid 169. ASO hybridization (Fig. 1) confirmed that resistant transfectants were expressing the transgene, with the signal dependent upon reverse transcription. Expression of the resistant transgene was stable over several weeks of continuous culture, although there was some variation in levels of expression between resistant transfectants derived from different electroporation experiments (Fig. 1). From an initial series of 11 independent resistant transfectants selected ( $3 \mathrm{R}$ to $18 \mathrm{R}$, Fig. la), lines $7 R$ and $17 R$ were selected as high expressers for functional analysis. To ensure clonality, 7R and $17 \mathrm{R}$ were recloned by limiting dilution: $7.1 \mathrm{R}$ to $7.12 \mathrm{R}$ were subcloned from $7 \mathrm{R}$; 17.1R to $17.5 \mathrm{R}$ from 17R. The ASO assays were repeated at intervals during the study to monitor continued expression of the transgene. Four independent susceptible transfectants (2S, 10S, 25S, 30S) were used as controls.

\section{Influence of Nramp on Respiratory Burst Activity}

Of the many pleiotropic effects associated with Lsh gene function, production of both ROI and reactive nitrogen intermediates (RNI) are directly implicated in the enhanced antimicrobial and tumoricidal activity of the resistant allele, and were thus selected as the first phenotypes to test the hypothesis that $N \mathrm{ramp}$ is $I t y / \mathrm{Lsh} / \mathrm{Bcg}$. Figure 2 shows the results of one experiment representative of five carried out in which resistant and susceptible transfectant clones were examined for PMA-elicited RB. Resting cells triggered with PMA produced substantial levels of ROI, with resistant transfectant clones consistently producing significantly more superoxide anion than susceptible transfectants (Fig. 2 a and c). In previous studies, LPS priming/activation has been shown to inhibit RB activity (24). Here, 24- or $30-\mathrm{hr}$ LPS $(25 \mathrm{ng} / \mathrm{ml}$ ) priming prior to PMA triggering extinguished ROI production in all susceptible transfectants (Fig. 2 b and d). In contrast, all resistant transfectants exhibited a persistence of RB at both 24 and $30 \mathrm{hr}$ poststimulation with LPS. Hence, sustained RB activity in LPS-treated macrophages provided a definitive phenotype for resistant transfectants compared with susceptible controls, suggesting a differential Nramp-mediated effect at the level of LPS priming.

\section{Influence of Nramp on Nitrite Production}

To elicit antimicrobial levels of NO, and hence nitrite release, by macrophages, both priming (e.g., LPS) and activation (e.g., IFN $\gamma$ ) stimuli are usually required (10). Hence, control cells and those treated with IFN $\gamma$ alone showed negligible nitrite release, although in some experiments a small amount was detectable in those treated with IFN $\gamma$ alone (not shown). LPS alone, or LPS plus IFN $\gamma$, elicited significantly higher levels of nitrite release in resistant transfectant clones compared with susceptible transfectants (Fig. 3). Clone $7.5 \mathrm{R}$ repeatedly and consistently produced the highest levels of ROI and nitrites among the 


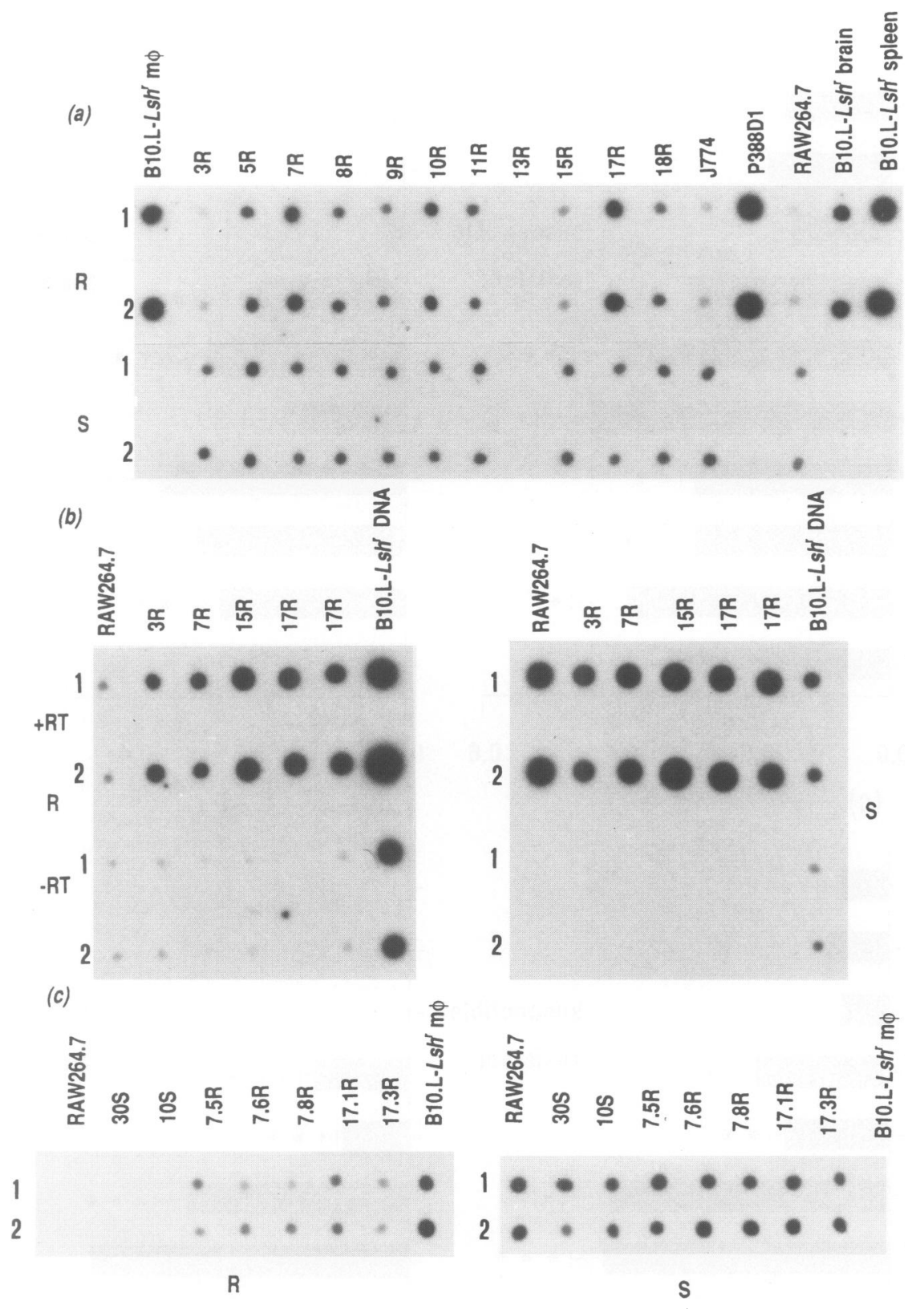

FIG. 1. Allele-specific oligonucleotide hybridization detects transgene expression in RAW 264.7 macrophages

RNA prepared from macrophages, brain or spleen tissues from Bl0.L-Lsh ${ }^{\mathrm{r}}$ congenic mice or RAW 264.7 transfectants following expansion were treated with DNAase, reverse transcribed and a specific Nramp fragment covering the susceptibility-associated mutation prepared by PCR. Aliquots of the PCRs were alkali-denatured, spotted in duplicate ( 1 and 2 ) onto membrane and probed with oligonucleotides corresponding to either the resistant (R) or susceptible (S) sequences. Posthybridization washing conditions were selected to achieve specificity for allelic forms. In Panel (a), a series of antibiotic transfectants clones were analyzed and a subset selected (b) to confirm that the resistant signal was derived from expressed RNA (+RT) rather than contaminating expression vector-derived DNA (-RT). As a positive resistant control, cDNA from B10.L-Lsh ${ }^{\mathrm{r}}$ macrophages was used, resulting in positive hybridization in the RT negative control. Subclones from the strongest expressing clones from Panel (a) were analysed in Panel (c), including several clones prepared with a susceptible allele Nramp expression construct. 
(a)

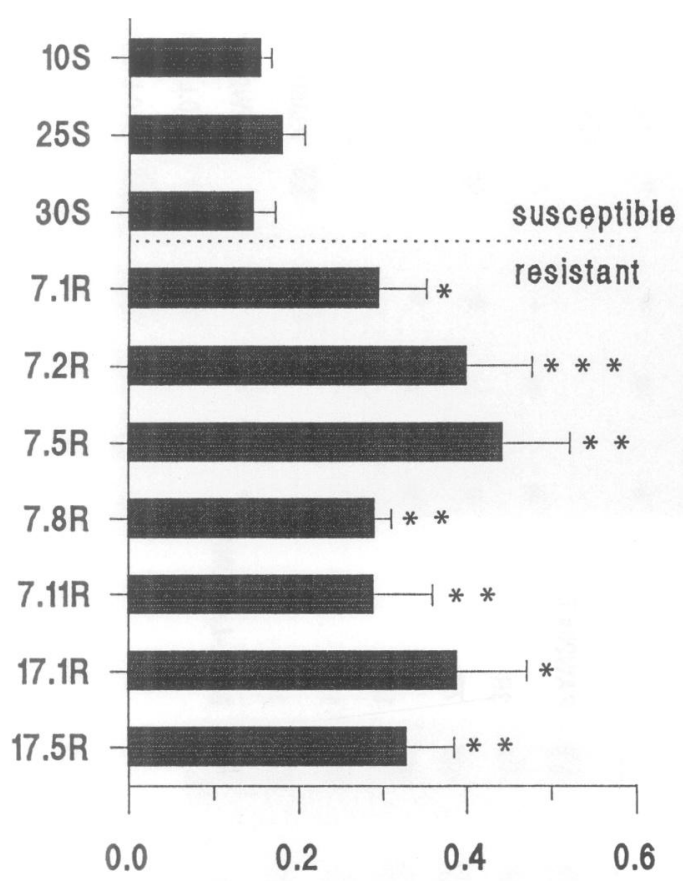

(c)



(b)

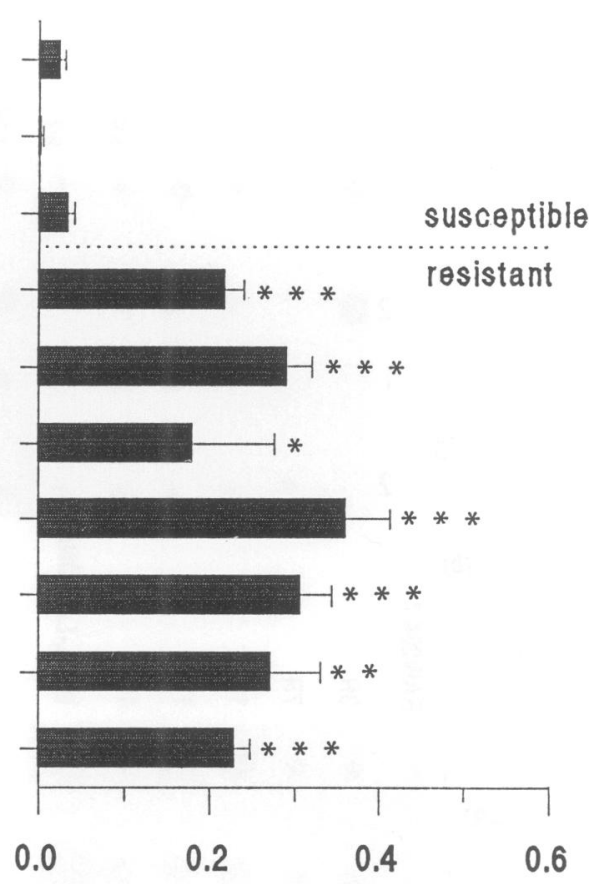

(d)

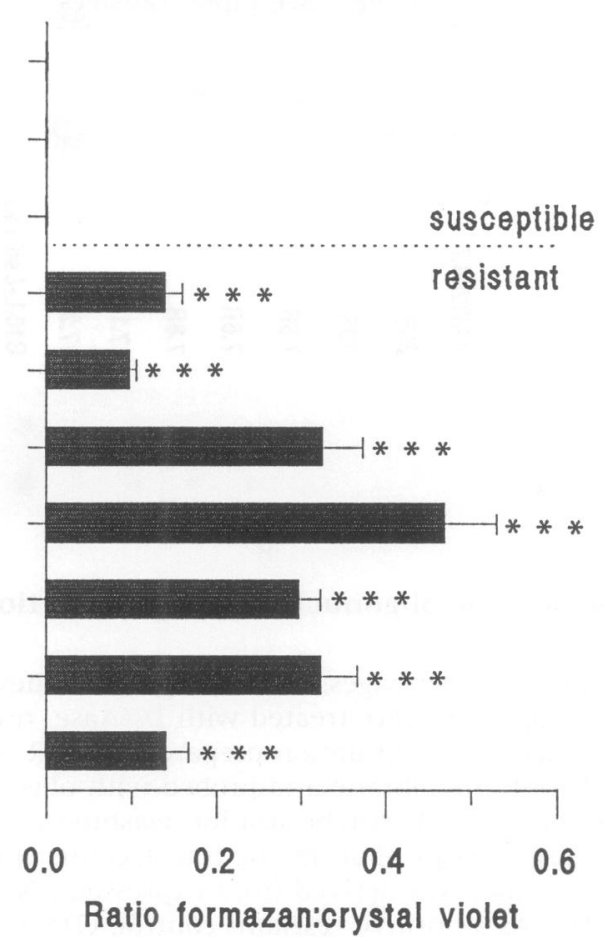

FIG. 2. Resistant allele RAW 264.7 transfectants generate enhanced RB responses, which are not extinguished following LPS stimulation

PMA-elicited RB was measured in resting ( $a$ and c) or LPS-treated (b and d) resistant and susceptible transfectants at 24 ( $a$ and b) or 30 (c and d) hr after LPS $(25 \mathrm{ng} / \mathrm{ml}$ ). Results are expressed as a ratio of formazan: crystal violet readings from six wells/treatment. Asterisks indicate significance levels $\left({ }^{*} p<0.05 ;{ }^{*} p<0.01\right.$; $\star \star \star p<0.001)$ for results of Student's $t$ tests used to compare each resistant transfectant against the susceptible transfectant 30S. Similar levels of significance were observed for comparisons with $10 \mathrm{~S}$ and $25 \mathrm{~S}$. Results are representative of five independent experiments performed. 


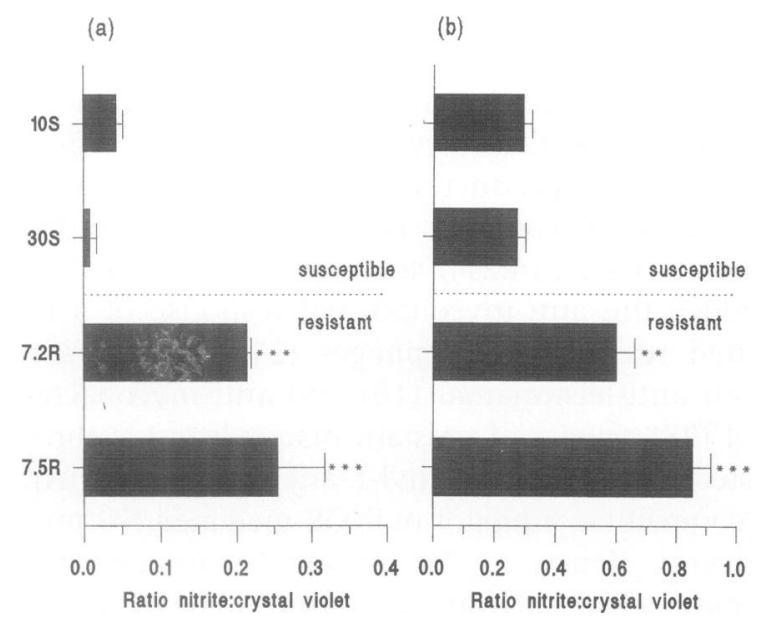

FIG. 3. Resistant allele RAW 264.7 transfectants exhibit enhanced NO generation in response to LPS (a) or IFN $\boldsymbol{\gamma}+$ LPS (b)

Cells were incubated for 24 or $30 \mathrm{hr}$ in the presence of LPS $(25 \mathrm{ng} / \mathrm{ml})$ or LPS plus IFN $\gamma(25 \mathrm{ng} / \mathrm{ml}$ and $25 \mathrm{U} / \mathrm{ml}$ ). Determinations were normalized to cell number from the crystal violet staining intensity of a parallel plate, and results are presented as the ratio of nitrite to crystal violet. Asterisks indicate significance levels $\left({ }^{*} p<0.05 ;{ }^{* *} p<0.01 ;{ }^{* *} p<0.001\right)$ for results of Student's $t$ tests used to compare each resistant transfectant against the susceptible transfectants $10 \mathrm{~S}$ and 30S. Clones 17.3R and 17.6R also showed significantly higher $(p<0.05)$ nitrite levels in this experiment. Results are representative of five independent experiments performed.

resistant transfectant clones, and was chosen for more detailed analysis of other Ity/Lsh/Bcgrelated phenotypes and for inhibitor studies.

\section{L-Arginine Uptake in Nramp Transfectant Clones}

L-Arginine is the substrate required for the generation of NO (and hence nitrite) via iNOS, and its uptake into macrophages, known as the $y^{+}$ phenotype, is regulated by activation (25). In previous studies (9), differences in uptake of Larginine into bone marrow-derived macrophages from congenic $L s h^{\mathrm{s}}$ and $L s h^{\mathrm{r}}$ mice were observed following activation with IFN $\gamma$, LPS, or IFN $\gamma$ plus LPS. Experiments were therefore performed to determine whether differential uptake of L-arginine in response to activation stimuli are detectable in Nramp-resistant and -susceptible transfectants. Results presented in Fig. 4 demonstrate that L-arginine uptake is up-regulated in resistant transfectants to a significantly greater degree than in susceptible transfectants in response to

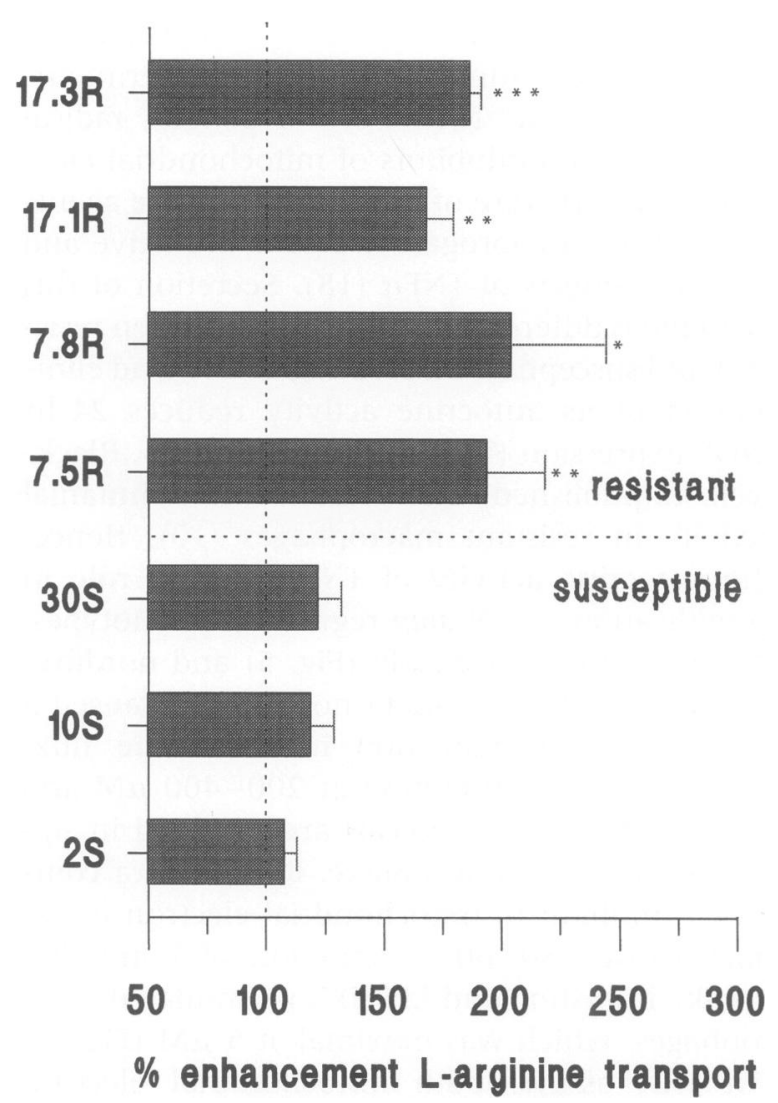

FIG. 4. L-Arginine uptake is enhanced in resistant compared with susceptible transfectants following activation with LPS plus IFN $\gamma$

Results are expressed as the percentage stimulation (standard deviation) observed in LPS + IFN $\gamma$-treated macrophages compared with untreated controls. Asterisks indicate significance levels $\left({ }^{*} p<0.05\right.$; $\left.{ }^{* *} p<0.01 ;{ }^{* *} p<0.001\right)$ for results of student's $t$ tests used to compare each resistant transfectant against the susceptible transfectants $2 \mathrm{~S}, 10 \mathrm{~S}$ and 30S. Results are representative of five independent experiments performed.

activation with IFN $\gamma$ plus LPS. Again, the 7.5Rresistant transfectant line gave the most consistent and reproducible results in at least five independent experiments, for which one representative experiment is shown. As with our previous experiments (9), there was a time-lag between stimulation and enhanced uptake of L-arginine from extracellular pools (data not shown), consistent with a requirement for transcriptional activation.

\section{Effect of Radical Scavengers and Mitochondrial Inhibitors on the Resistance Phenotype}

In order to identify candidate signaling pathways used to achieve the Ity/Lsh/Bcg-regulated pleio- 
tropic effects now confirmed in Nramp-resistant transfectants, a number of inhibitor experiments were performed. The effect of general radical scavengers and inhibitors of mitochondrial electron transport were of interest since these agents were shown to abrogate the gene inductive and cytotoxic effects of TNF $\alpha$ (18). Secretion of this cytokine is differentially regulated between resistant and susceptible macrophages (26), and elimination of its autocrine activity reduces $24-\mathrm{hr}$ iNOS expression (T. I. A. Roach and J. M. Blackwell, unpublished) and inhibits antileishmanial activity in resistant macrophages (10). Hence, the autocrine activity of TNF $\boldsymbol{\alpha}$ plays a role in amplification of Nramp-regulated phenotypes. Butylated hydroxyanisole (Fig. 5) and nordihydroxyguaraietic acid (data not shown) caused a dose-dependent reduction in L-arginine flux, showing $>80 \%$ inhibition at $200-400 \mu \mathrm{M}$ and indicating that free radicals are involved in signaling for L-arginine uptake. Rotenone, a complex I inhibitor of mitochondrial electron transport, caused $30-60 \%$ inhibition of L-arginine uptake in resting and LPS/IFN $\gamma$-stimulated macrophages, which was maximal at $5 \mu \mathrm{M}$ (Fig. 5). For ROI assays, both mitochondrial electron transport inhibitors rotenone and thenoyltrifluoroacetone caused dose-dependent inhibition in control and LPS + IFN $\gamma$-stimulated macrophages, which was maximal at the highest $(40$ or $400 \mu \mathrm{M})$ concentration of each inhibitor (Fig. 6). These results suggest a role for mitochondrially derived ROI in signaling for the Nramp-regulated macrophage activation phenotypes L-arginine uptake and RB.

\section{DISCUSSION}

Functional analysis of three independent macrophage activation phenotypes, $\mathrm{RB}$, nitrite release, and L-arginine transport, in susceptible macrophages transfected with the resistant allele provides evidence that the product of the Nramp gene, as expressed within the bone marrow-derived macrophage (16), encodes Ity/Lsh/Bcg. This is supported by our independent observation (T. Lang, C. H. Barton, and J. M. Blackwell, unpublished) that Nramp-resistant transfectants show enhanced class II expression in response to IFN $\gamma$ and are more efficient at presenting defined recombinant antigen to a Leishmania-specific $\mathrm{T}$ cell clone. Together these observations provide the first compelling functional evidence that Nramp is $I t y / L s h / B c g$. Two of the phenotypes ex- amined here, RB and NO production, are of particular importance since both are implicated in macrophage antimicrobial activity. Previous studies suggest, however, that although differences in ROI production are observed in macrophages from congenic resistant and susceptible mouse strains $(4,27)$, scavengers of ROI do not reduce the anti-mycobacterial response of activated resistant macrophages (27). In contrast, both anti-leishmanial (10) and anti-mycobacterial (28) activity of resistant macrophages is abrogated by $\mathrm{N}^{\mathrm{G}}$-monomethyl-L-arginine (L-NMMA), a competitive inhibitor of iNOS-mediated NO production. Hence, in the murine Nramp-regulated antimicrobial pathway, NO appears to be the major final effector molecule for antimicrobial activity. This may, however, have more to do with the sensitivity of the target to ROI versus RNI, rather than that the Nramp-regulated RB is ineffectual. In human macrophages, where iNOS-generated NO does not appear to play a major role in antimicrobial activity (29), differential Nramp-regulated RB responses may play a more prominant role in determining an antimicrobial/tumouricidal phenotype. The importance of this pathway in human antimicrobial defence is well evidenced by the severe susceptibility to infectious agents observed in chronic granulomatous disease patients. These patients fail to mount an NADPH oxidase-dependent respiratory burst response, due to a series of complementary mutations in the polypeptide chains which make up the oxidase complex (30).

The generation of high levels of NO for antimicrobial activity is dependent on transcriptional regulation of iNOS expression (reviewed Ref. 31), and may be limited by L-arginine flux (25). Previous studies have demonstrated differential up-regulation of iNOS mRNA in response to IFN $\gamma$ in macrophage lines derived from $B c g$ congenic mouse strains (28). In our experiments, resting RAW 264.7 transfectants produced negligible NO, and required LPS activation. LPS alone was sufficient to induce enhanced levels of nitrite release from some Nramp-resistant transfectants compared with susceptible controls, although this measure of resistance was not as strong as ROI production. IFN $\gamma$ acted synergistically with LPS, again resulting in significantly higher nitrite release in resistant compared with susceptible transfectants. Although we did not analyze iNOS message in Nramp transfectant clones, these results are consistent with recent analysis of iNOS promoter region demonstrating functional elements responsible for LPS inducibility and IFN $\gamma$ synergism (32), and suggest that 
(a)

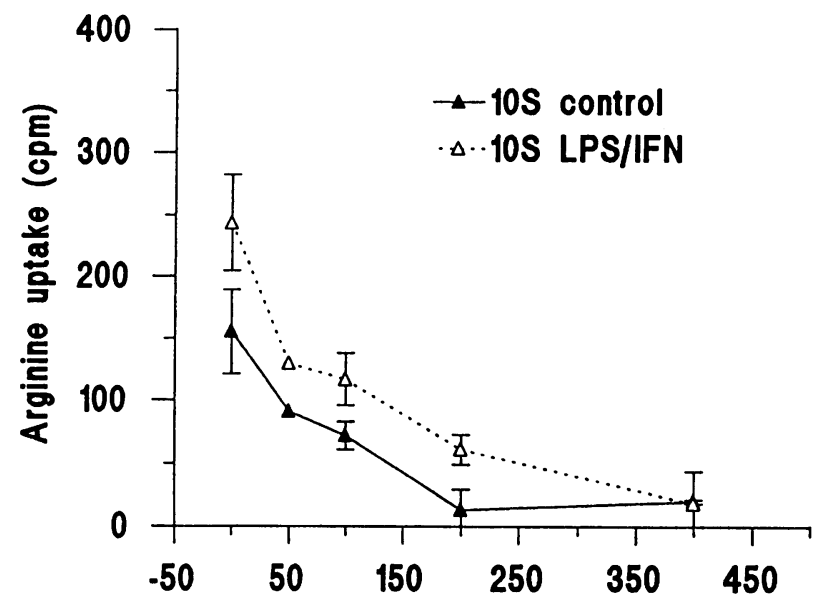

(b)

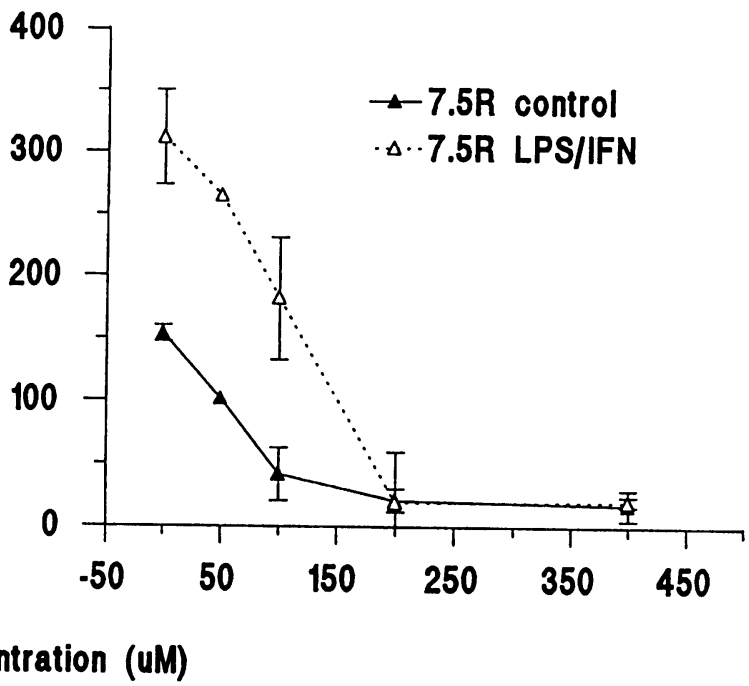

(c)

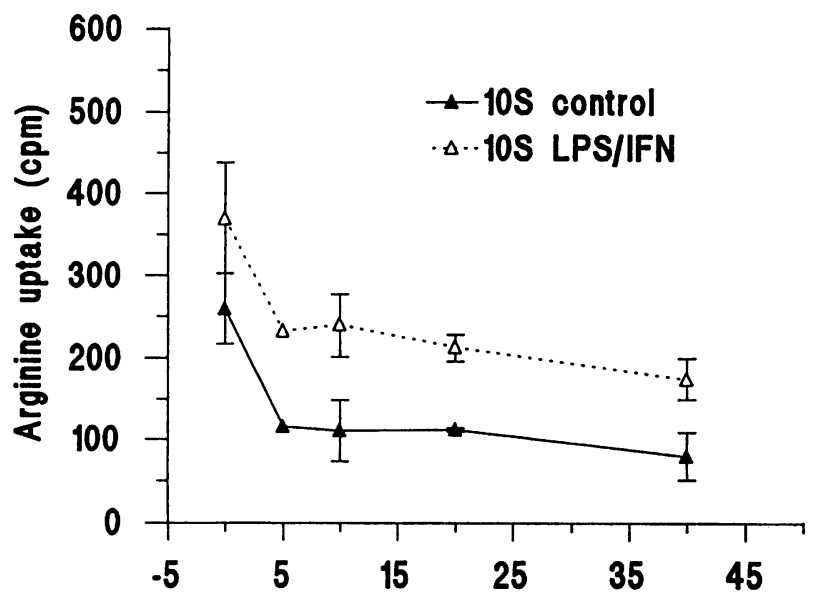

(d)



Rotenone concentration (UM)

FIG. 5. L-Arginine uptake is inhibited by agents that suppress the production of radicals

Resting or LPS/IFN $\gamma$-treated resistant ( $b$ and $d$ ) and susceptible ( $a$ and $c$ ) transfectants were pretreated with the radical scavenger BHA ( $a$ and $b$ ) or the mitochondrial electron transport inhibitor rotenone (c and d) at the doses indicated. Inhibitor was added $1 \mathrm{hr}$ after plating and prior to the addition of media alone or that containing LPS $(25 \mathrm{ng} / \mathrm{ml})+\operatorname{IFN} \gamma(25 \mathrm{U} / \mathrm{ml})$. As before, enhanced stimulated L-arginine flux was observed in resistant versus susceptible macrophages. Treatment with BHA (NDHGA data not shown, but similar effects) or rotenone caused a dose-dependent decrease in the uptake of L-arginine in both resting (filled) and activated (open) cells.

Nramp may play a part in regulating both of these gene inductive events for iNOS expression.

of parallel importance in determining the NO antimicrobial phenotype may be our demonstration that Nramp-resistant transfectants show enhanced L-arginine uptake following LPS/IFN $\gamma$ activation compared with susceptible transfec- tant controls. The time course for induction of enhanced L-arginine transport with activation was consistent with that reported for other murine macrophages (25), suggesting a requirement for transcriptional regulation of expression of the transporter molecule involved. Although we considered the possibility that Nramp itself may 


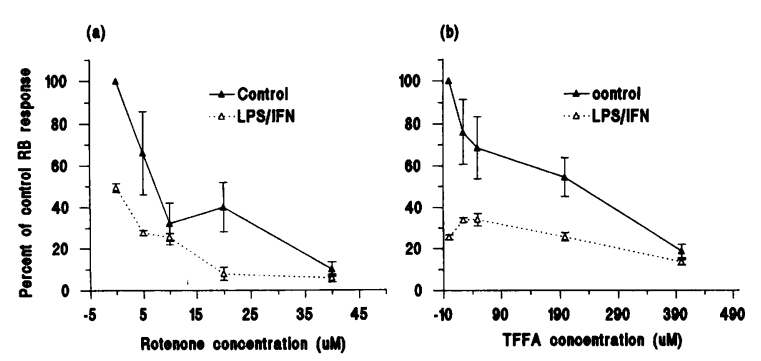

FIG. 6. Inhibition of mitochondrial electron transport suppresses RB in RAW 264.7 transfectant macrophages

Resistant allele transfectant clone $7.5 \mathrm{R}$ was used for inhibition studies with the complex I inhibitor rotenone (a) and the complex II inhibitor thenoyltrifluoroacetone (b). Control (filled) and activated cells (open) exhibited a dose-dependent decrease in ROI production with both drugs.

be an L-arginine transporter (9), no dramatic increases in Nramp expression at the mRNA level are observed (16) with LPS/IFN $\gamma$ activation over the time period necessary to achieve the LPS/ IFN $\gamma$-induced increase in L-arginine uptake. Hence, regulation of L-arginine uptake appears to be another pleiotropic effect of the Nramp gene involving its influence in LPS/IFN $\gamma$ gene inductive pathways. Recent studies (33) have demonstrated that LPS-activated RAW 264.7 macrophages express a third member, MCAT$2 \mathrm{~B}$, of the mouse cationic amino acid transporters (MCAT) family of cationic amino acid transporters, which is responsible for enhanced L-arginine influx following activation. Studies are in progress to examine MCAT-2B mRNA expression in RAW 264.7 Nramp transfectants following LPS/IFN $\gamma$ activation.

Our demonstration that Nramp transfection influences a variety of phenotypes associated with macrophage activation and antimicrobial activity are consistent with the many studies (reviewed Refs. 5, 6, and 9) carried out with macrophage populations isolated from chromosome 1 congenic mouse strains in which the Ity/Lsh/ $B c g$-resistant allele has been bred onto a susceptible (BALB or B 10) genetic background. All suggest that the gene acts at some key point early in the macrophage priming/activation pathway, possibly at the level of signal transduction. In our continuing attempts to pinpoint the site of action of the gene, we became interested $(9,13)$ in the possibility that the generation of free radicals (ROI or RNI) might play an important part in signaling, as well as being the effector molecules for antimicrobial or tumoricidal activity. In the present study, we were motivated to re-examine the role of free radicals in Nramp-regulated gene induction by two important observations: (1) that murine and human Nramp sequences show $53-58 \%$ similarity with two yeast mitochondrial proteins, SMF1 and SMF2 $(9,17)$; and (2) that mitochondrial generation of ROI is important in the cytotoxic and gene inductive effects of TNF $\alpha$ (18). Using the $\mathrm{y}^{+}$phenotype as a readout for Nramp-regulated gene induction, initial experiments using broad-specificity free radical scavengers confirm that radical generation is important for L-arginine transport following LPS/IFN $\gamma$ activation and in resting cells. The further demonstration that rotenone, which inhibits electron entry from complex I to ubiquinone during mitochondrial electron transport, also inhibits LPSinduced and resting $\mathrm{y}^{+}$phenotype provides support for the hypothesis that mitochondrial generation of ROI is important in Nramp-regulated intracellular signaling events. In the case of $\mathrm{TNF} \alpha$-induced changes in gene expression, inhibition of mitochondrial electron transport has been shown to specifically inhibit activation of the NF $\kappa B$ transcription factor (18). Since the gene inductive effects of LPS are also mediated through activation of $\mathrm{NF} \kappa \mathrm{B}(34,35)$, a role for Nramp in modulating mitochondrial activity, and generation of ROI via the electron transport system for activation of $\mathrm{NF} \kappa \mathrm{B}$, might provide a common mechanism for many of the Nramp-regulated LPS-induced pleiotropic effects on macrophage priming/activation pathways. Studies are in progress to determine whether mitochondrial inhibitors modulate LPS- or TNF $\alpha$-induced activation of NF $\kappa$ B in Nramp-resistant and -susceptible transfectants.

A requirement for mitochondrially derived ROI in intracellular signaling provides an interesting hypothesis to account for the many gene inductive events (e.g., up-regulation of KC, IL-1, TNF $\alpha$, iNOS, MHC class II; reviewed in Ref. 5, 6, and 9) which appear to be differentially regulated in Nramp-resistant versus -susceptible macrophage populations. However, not all of these require activation of the NF $\kappa \mathrm{B}$ transcription factor, implying that mitochondrial generation of ROI might be important in other signaling pathways. PMA-elicited RB response is primed in resting RAW 264.7 cells, and is immediately enhanced in Nramp-resistant allele transfectants. $\mathrm{RB}$ activity is dependent upon assembly of the NADPH oxidase complex (reviewed Ref. 31) at the phagocyte membrane, involving translocation of two cytosolic components, p47phox and 
p67phox, to membrane bound subunits, gp9lphox and p22phox, of the cytochrome ${ }_{b 558}$. PMA activates protein kinase $C$, which is required for phosphorylation of $\mathrm{p} 47 \mathrm{phox}$ prior to translocation to the membrane. However, PMA does not itself induce the translocation event. In our studies, inhibition of mitochondrial electron transport with rotenone or thenoyltrifluoroacetone also inhibited PMA-elicited RB, suggesting that the mitochondrial generation of ROI signals for membrane assembly of the NADPH oxidase complex. As with other studies (24), LPS proved to be a potent inhibitor of the RB response. Interestingly, the degree of inhibition was markedly less in all Nramp resistant compared with susceptible transfectants, allowing the resistant transfectant to retain high levels of RB activity following LPS/IFN $\gamma$ activation. The sustained ability in resistant macrophages to produce ROI following LPS treatment would allow for the cogeneration of NO and superoxide anion within the same cell and hence rapid, diffusion-controlled production of the stable and highly toxic peroxynitrite anion (36).

Overall, these results provide an intriguing link between murine Nramp and mitochondrial function, in addition to the sequence similarity between Nramp and the yeast mitochondrial proteins SMF1 and SMF2. The molecular mechanism of Nramp and its influences over the mitochondrial generation of ROI remains unclear, but experiments are in progress to determine whether Nramp can complement the activities of SMF1/SMF2 in yeast knockouts (37). It will also be of interest to determine whether other Nramp-related sequences (e.g. Ref. 38) represent more ubiquitously expressed homologs to the yeast genes. Of particular interest is the observation that the putative $\mathrm{SH} 3$ binding domain at the $\mathrm{NH}_{2}$ terminus of murine $(16)$ and human $(17,39)$ Nramp genes is not duplicated in yeast, suggesting that this addition is related to its macrophage function. This domain, in particular, may be implicated in signaling via receptor-associated protein tyrosine kinases following receptor ligation, a pathway shown to mediate differential Nrampregulated pleiotropic effects on macrophage function (26). Direct interaction of this domain with protein tyrosine kinases may regulate transport function. Clearly, these are exciting times both for the structural and functional analysis of the Nramp gene family, and for the identification of other genes operating in this macrophage restricted signaling pathway leading to NF $\kappa \mathrm{B}-\mathrm{me}$ diated and other gene inductive events.

\section{ACKNOWLEDGMENTS}

This work was supported by grants from the Wellcome Trust.

\section{REFERENCES}

1. Plant J, Glynn AA. (1974) Natural resistance to Salmonella infection, delayed hypersensitivity and Ir genes in different strains of mice. Nature 248: 345-347.

2. Bradley DJ. (1974) Genetic control of natural resistance to Leishmania donovani. Nature 250: 353-354.

3. Skamene E, Gros P, Forget A, Kongshavn PAL, St Charles C, Taylor BA. (1982) Genetic regulation of resistance to intracellular pathogens. Nature 297: 506-509.

4. Blackwell JM, Toole S, King M, Dawda P, Roach TI, Cooper A. (1988) Analysis of Lsh gene expression in congenic B10.L-Lshr mice. Curr. Top. Microbiol. Immunol. 137: 301309.

5. Blackwell JM. (1989) (Convenor, 27th Forum in Immunology) The macrophage resistance gene Lsh/Ity/Bcg. Res. Immunol. 140: 767-828.

6. Blackwell JM, Roach TIA, Atkinson SE, Ajioka JW, Barton CH, Shaw M-A. (1991) Genetic regulation of macrophage priming/ activation: the Lsh gene story. Immunol. Lett. 30: 241-248.

7. Schurr E, Radzioch D, Malo D, Gros P, Skamene E. (1991) Molecular genetics of inherited susceptibility to intracellular parasites. Behring. Inst. Mitt. 88: 1-12.

8. Schurr E, Malo D, Radzioch D, et al. (1991) Genetic control of innate resistance to mycobacterial infections. Immunol. Today 12: A42-A45.

9. Blackwell JM, Barton $\mathrm{CH}$, White $\mathrm{JK}$, et al. (1994) Genetic regulation of leishmanial and mycobacterial infections: The Ity/Lsh/Bcg gene story continues. Immunol. Lett. 43: 99107.

10. Roach TI, Kiderlen AF, Blackwell JM. (1991) Role of inorganic nitrogen oxides and tumor necrosis factor-alpha in killing Leishmania donovani amastigotes in gamma interferonlipopolysaccharide-activated macrophages from $L s h^{\mathrm{s}}$ and $L s h^{\mathrm{r}}$ congenic mouse strains. Infect. Immun. 59: 3935-3944.

11. Kaye PM, Blackwell JM. (1989) Lsh, antigen 
presentation and the development of CMI. Res. Immunol. 140: 810-815.

12. Bradley DJ, Kirkley J. (1977) Regulation of Leishmania populations within the host. I. The variable course of Leishmania donovani infections in mice. Clin. Exp. Immunol. 30: 119-129.

13. Roach TIA, Chatterjee D, Blackwell JM. (1994) Induction of early response genes KC and JE by mycobacterial lipoarabinomannans: Regulation of $\mathrm{KC}$ expression in murine macrophages by Ity/Lsh/Bcg (candidate Nramp). Infect. Immun. 62: 1176-1184.

14. Vidal SM, Malo D, Vogan K, Skamene E, Gros P. (1993) Natural resistance to infection with intracellular parasites: Isolation of a candidate for Bcg. Cell 73:469-485.

15. Malo D, Vogan K, Vidal S, et al. (1994) Haplotype mapping and sequence analysis of the mouse Nramp gene predict susceptibility to infection with intracellular parasites. Genomics 23:51-61.

16. Barton $\mathrm{CH}$, White JK, Roach TIA, Blackwell JM. (1994) $\mathrm{NH}_{2}$-terminal sequence of macrophage-expressed natural resistance-associated macrophage protein (Nramp) encodes a proline/serine-rich putative $\mathrm{Src}$ homology 3-binding domain. J. Exp. Med. 179: 16831687.

17. Blackwell JM, Barton $\mathrm{CH}$, White JK, et al. (1995) Genomic organization and sequence of the human NRAMP gene: Identification and mapping of a promoter region polymorphism. Mol. Med. 1: 194-205.

18. Schulze-Osthoff $K$, Beyaert R, Vandevoorde V, Haegeman G, Fiers W. (1993) Depletion of the mitochondrial electron transport abrogates the cytotoxic and gene-inductive effects of TNF. E.M.B.O. J. 12: 3095-3104.

19. Morgenstern JP, Land H. (1990) Advanced mammalian gene transfer: High titre retroviral vectors with multiple drug selection markers and a complementary helper-free packaging cell line. Nucleic Acids Res. 18: 3587-3596.

20. Raschke WC, Baird S, Ralph R, Nakoinz I. (1978) Functional macrophage cell lines transformed by Abelson leukemia virus. Cell 15: $261-267$.

21. Stacey KJ, Ross IL, Hume DA. (1993) Electroporation and DNA-dependent cell death in murine macrophages. Immunol. Cell Biol. 71: 75-85.

22. Rook GAW, Steele J, Umar S, Dockrell HM. (1985) A simple method for the solubilisa- tion of reduced NBT, and its use as a colorimetric assay for activation of human macrophages with gamma-interferon. J. Immunol. Methods 82: 161.

23. Bradley DJ. (1977) Regulation of Leishmania populations within the host. II. Genetic control of acute susceptibility of mice to Leishmania donovani infection. Clin. Exp. Immunol. 30: $130-140$.

24. Ding AH, Nathan CF. (1987) Trace levels of bacterial lipopolysaccharide prevent interferon-gamma or tumor necrosis factor- $\alpha$ from enhancing mouse peritoneal macrophage respiratory burst capacity. J. Immunol. 139: 1971-1977.

25. Bogle RG, Baydoun AR, Pearson JD, Moncada S, Mann GE. (1992) L-arginine transport is increased in macrophages generating nitric oxide. Biochem. J. 284: 15-18.

26. Formica S, Roach TIA, Blackwell JM. (1994) Interaction with extracellular matrix proteins influences Ity/Lsh/Bcg (candidate Nramp) gene regulation of macrophage priming/activation for tumour necrosis factor $\alpha$ and nitrite release. Immunology 82: 4250.

27. Denis M, Forget A, Pelletier M, Skamene E. (1988) Pleiotropic effects of the $B c g$ gene: III. Respiratory burst in $B c g$-congenic macrophages. Clin. Exp. Immunol. 73: 370.

28. Barrera LF, Kramnik I, Skamene E, Radzioch D. (1994) Nitrite production by macrophages derived from $\mathrm{Bcg}$-resistant and susceptible congenic mouse strains in response to interferon-gamma and infection with BCG. Immunology 82: 457.

29. Schneeman M, Schoedon G, Hofer S, Blau N, Guerrero L, Schaffer A. (1993) Nitric oxide is not a constituent of the antimicrobial armature of human mononuclear phagocytes. J. Infect. Dis. 167: 1358-1363.

30. Roos D. (1994) The genetic basis of chronic granulomatous disease. Immunol. Rev. 138: 121-157.

31. Bastian NR, Hibbs Jr JB. (1994) Assembly and regulation of NADPH oxidase and nitric oxide synthase. Curr. Opin. Immunol. 6: 131139.

32. Martin E, Nathan C, Xie Q-W. (1994) Role of interferon regulatory factor $\mathrm{I}$ in induction of nitric oxide synthase. J. Exp. Med. 180: 977984.

33. Closs EI, Lyons CR, Kelly C, Cunningham JM. (1993) Characterization of the third member of the MCAT family of cationic 
amino acid transporters. J. Biol. Chem. 268: 20796-20800.

34. Vincenti MP, Burrell TA, Taffet SM. (1992) Regulation of NF-kappaB activity in murine macrophages. Effect of bacterial lipopolysaccharide and phorbol ester. J. Cell Physiol. 150: 204-213.

35. Zheng S, Brown MC, Taffet SM. (1993) Lipopolysaccharide stimulates both nuclear localization of the nuclear factor kappa $B$ 50k-Da subunit and loss of the 105-kDa precursor in RAW264 macrophage-like cells. $J$. Biol. Chem. 268: 17233-17239.

36. Beckman JS, Beckman TW, Chen J, Marshall PA, Freeman BA. (1990) Apparent hydroxyl radical production by peroxynitrate: Implications for endothelial injury from ni- tric oxide and superoxide. Proc. Natl. Acad. Sci. U.S.A. 87: 1620-1624.

37. West AH, Clark DJ, Martin J, Neupert W, Hartl FU, Horwich AL. (1992) Two related genes encoding extremely hydrophobic proteins suppress a lethal mutation in the yeast mitochondrial processing enhancing protein. J. Biol. Chem. 267: 24625-24633.

38. Dosik JK, Barton CH, Holiday DL, Krall MM, Blackwell JM, Mock BA. (1994) An Nramprelated sequence maps to mouse chromosome 17. Mammalian Genome 5: 458-460.

39. White JK, Shaw M-A, Barton $\mathrm{CH}$, et al. (1994) Genetic and physical mapping of $2 q 35$ in the region of NRAMP and IL8R genes: Identification of a polymorphic repeat in exon 2 of NRAMP. Genomics 24: 295-302.

Contributed by K. S. Warren on January 23, 1995. 